\title{
CardNutri: A software of Weekly Menus Nutritional Elaboration for Schoolar Feeding applying Evolutionary Computation
}

\author{
Rafaela P. C. Moreira ${ }^{1} \bowtie$, Elizabeth F. Wanner ${ }^{2}$ 凶, Flávio V. C. Martins ${ }^{3}$ 凶, \\ and João F. M. Sarubbi ${ }^{3} \bowtie$ \\ ${ }^{1}$ Programa de Pós-Graduação em Modelagem Matemática e Computacional CEFET, \\ MG, Brazil \\ rafapcmor@gmail.com $\odot$ \\ 2 ALICE Group - EAS, Aston University, UK \\ e.wanner@aston.ac.uk \\ ${ }^{3}$ Departamento de Computação, CEFET, MG, Brazil \\ flaviocruzeiro@decom. cefetmg.br @ joaosarubbi@gmail.com $\mid$
}

\begin{abstract}
This paper aims to present and evaluate a software that uses an evolutionary strategy to design weekly nutritional menus for School Feeding. The software ensures the nutritional needs of students and also minimizes the total cost of the menu. We based our nutritional needs on the Brazilian National School Feeding Programme (PNAE). This program takes into account: (i) the age of the student; (ii) some preparations issues as color, consistency and, variety; and also (iii) the maximum amount to be paid per meal. Our software generates, in less than five minutes, a set of menus, and the nutritionist can choose the menu that suits his/her best. We evaluate our algorithm using the Weighted-Sum approach, and our results show that the obtained 5-days menus using the proposed methodology not only comply with the restrictions imposed by the authorities but also produce inexpensive and healthy menus. We also appraise the software itself using an opinion pool among nine nutritionists. The professionals considered our software above expectations.
\end{abstract}

Keywords: Evolutionary Computation; Food Technology; Public Health Informatics; Menu Planning; School Feeding.

\section{Introduction}

Society has always been concerned about feeding since an inadequate nutrition is responsible for the growth and health problems in human beings. With the new technologies' development, not only the food production has grown but also has the industrial food accessibility. An inadequate nutrition with a combination of high levels of sugar and fat presented in the industrialized food can also lead to obesity or overweight.

Food is an essential condition for life maintenance. However, it has to be ingested in a proper quantity and variety. Overall functional consequences of a 
malnourished individual are enormous: impairment in growth, intellectual performance and in women reproductive capacity, to name a few.

The eating guide for the Brazilian population 2 presents a broad definition of healthy eating: "An adequate and healthy eating is a fundamental human right that guarantees access to an eating practice appropriate to the biological and social aspects of the individual and which must comply with special dietary needs. It must be referenced by social eating habits, gender, race, and ethnicity; it must be accessible from a physical and financial point of view; it must have a balance between quantity and quality while ensuring the principles of variety, balance, moderation and taste".

The practice of healthy eating has become important since childhood, at which stage eating habits are formed, so the family and school environment contribute to the knowledge of new foods.

In this way, this diet must supply energy, macronutrients, and micronutrients intake levels enough to meet the daily nutrient requirements of students.

In Brazil, there is a program that regulates the school feeding, called the Brazilian National School Feeding Programme (PNAE) [5]. This program aimed to attend children in darycare, preschool, elementary school, middle school and high school.

This program assures that there is a nutritionist responsible for the elaboration of the school menu in the executing entities was required. The goal was to provide a balanced diet to the students, improving the quality of the PNAE, since this activity was carried out by the school cooks. The nutritionist is responsible for school meals, preparation of the menu as well as monitoring the production of meals in schools.

The general purpose of Menu Planning is to achieve balanced and economical meals to satisfy a set of simultaneous requirements. The problem aims to find the best combination of items that meet the stipulated goals and requirements [1.

In this work, we present CardNutri, a software that uses a evolutionary strategy to design weekly nutritional menus for School Feeding. The software considers two fundamental aspects: (i) the cost that must be reduced and, (ii) the student's daily nutritional needs that must be achieved. Desirable properties such as: nutritional reference according to the age range provided by PNAE, variety, harmony of the culinary preparations and a financial cost limit for each type of meal are inserted in the problem as requirements constraints. The software uses a multiobjective approach considering the two aspects: (i) cost; and, (ii) nutritional reference.

The contributions of this work are twofold. First, a set of 5-day menus is obtained using the Weighted-Sum approach. The results show that the menus not only comply with the restrictions imposed by the Brazilian authorities but also are healthy and inexpensive menus. Secondly, the software is evaluated using an opinion pool among nine nutritionists to assess the usability and adequability issues. The professionals consider the software very useful and above expectations. 
This paper is organized as follows: Section 2 presents related works. Section 3 presents the problem definition. Section 4 describes the applied algorithm. Section 5 shows the developed application. Section 6 describes the results. And, finally, Section 7 concludes the work.

\section{Related Works}

Since 1964, computer-aid techniques have been used to solve the Menu Planning Problem (MPP). The work of Balintfy [1] used Linear Programming (LP) techniques to construct the first menu planner, to find the minimum cost and to comply with the daily nutritional needs with a desired degree of variety, taking into account color and consistency requirements for a specific number of days. The proposed algorithm was applied in a hospital and was able to reduce up to $30 \%$ of the menu cost.

Kahraman and Seven [10] formulated a bi-objective diet problem and solved it applying the Linear Scalarization approach using a mono-objective Genetic Algorithm. The objectives were: to minimize the cost and to maximize the rating, according to the user's preference.

The Departments of Nutrition and Computer Science at the University of Sonora in Mexico, in 2007, developed a computer system PlaDiet, aiming to calculate individual meals, attending all the nutritional requirements established by a professional. A non-linear integer programming model was constructed, using Genetic Algorithms to solve it. The program allows to choose the type of meals (lunch, dinner, and intermediates), making available, in seconds, diets for any caloric need within 28 days 8 .

In 2012, other software, Pro-Diet 9], proposes the prescription of menus and diet formulation, to meet the principles of healthy eating, taking into account the colors and meals variety, the combination of flavors, the texture and the provision of all nutrients. The software also used a Genetic Algorithm and can generate menus in seconds, which could, manually, take hours.

A model of Menu Planning for secondary schools in Malaysia using Integer Programming was proposed by Suliad and Ismail [15]. The aim was to maximize the variety of nutrient and to minimize the cost.

It is worthwhile to notice that our work differs from the others available in the literature. In the first place, the main goal is to produce a set of collective menus ensuring that some requirements are followed, such as color, variety, harmony. Furthermore, the menus need to be healthy and under a threshold value. In the second place, and not least important, a simple and easy-to-use software is presented. The software has some additional features making it very appealing for the nutritionists and reducing considerably the time spent in the Menu Planning. 


\section{Problem Definition}

In this Section, we introduce the basic concepts to understand the problem and how model the MPP. We want to create a 5-day menu to meet the nutritional needs of full-time 4 to 5 -year old students according to the nutriotional reference of the PNAE.

Students in a full-time school day must get not less than three meals representing at least $70 \%$ of their daily nutritional needs. Table 1 shows the recommended values for $70 \%$ of daily nutritional needs (DNN) for preschool students [5]. In this table, CHO refers to carbohydrates, PTN to proteins and LIP to lipids. Other references can be found in [5]. These references were based to Food and Agriculture Organization of United Nations (FAO) [13.

Table 1: Reference values for energy, macro and micro nutrients for preschool (4 to 5 years)- $70 \%$ DNN

\begin{tabular}{|l|l|l|l|l|l|l|l|l|}
\hline Energy & CHO & PTN & LIP & Fibers & \multicolumn{4}{|l|}{ Minerals $(\mathrm{mg})$} \\
\hline (Kcal) & $(\mathrm{g})$ & $\mathrm{g})$ & $\mathrm{g})$ & $\mathrm{g})$ & $\mathrm{Ca}$ & $\mathrm{Fe}$ & $\mathrm{Mg}$ & $\mathrm{Zn}$ \\
\hline 950.00 & 154.40 & 29.70 & 23.80 & 17.50 & 560.00 & 7.00 & 91.00 & 3.50 \\
\hline
\end{tabular}

In this work, each day is composed of three meals: (i) breakfast; (ii) lunch; and, (iii) afternoon snack. Each meal has some preparations, according to its type. The breakfast is composed of three preparations: (i) bread or other cereal; (ii) milk or derivatives; and, (iii) fruit. The lunch is composed of seven preparations: (i) rice; (ii) beans; (iii) entree; (iv) side dish; (v) main dish; (vi) dessert: fruit or other; and, (vii) juice. The snack is composed of three preparations: (i) bread or other cereal; (ii) drink (milk or derivatives or juice); and, (iii) fruit.

We defined two objective functions and there is a set of constraints to be met. The first objective function aims to minimize the nutritional error, thus meeting the nutritional needs value. This function, based on the work proposed by $[9$, calculates the absolute difference of the sum of the nutrients and the sum of established reference. In this work, we use the established reference by PNAE. The second objective function aims to minimize the menu cost.

The principle of PNAE is to promote healthy and adequate school meals in accordance to the age group, among other characteristics. Given this, in this work, some constraints are adopted for the PNAE principles to be attended: (i) color; (ii) consistency, and (iii) variety. We also implemented other constraints: (i) cost limit for each meal for each student; (ii) nutritional error limit. The color, consistency, and variety constraints are related to the nutritional error function and, the cost limit constraint is related to the cost function. The constraints of the problem are described below:

- Color: A diversified meal in color ensures a balanced and nutrient-rich dietary. For this work, four predominant colors were defined: (i) yellow; (ii) red; (iii) green; and, (iv) brown. This constraint is checked only for lunch and 
for the following types of preparations: (i) entree; (ii) side dish; (iii) dessert; and, (iv) drink, as directed by [9. To ensure this color diversification, the number of repetition of the same color in a given meal should be less than or equal to 2.

- Consistency: A good texture transmits confidence in the quality and acceptability of food. The consistency is classified into 2 categories: (i) liquid/pasty; and, (ii) semi-solid/solid. It is not allowed to have more than one type of preparations classified as liquid/pasty in the lunch. The preparations checked are: (i) beans; (ii) side dish; and, (iii) main dish.

- Variety: The food variety is indispensable for all vital nutrients that can be supplied. To ensure that meals are varied, we checked the number of repeated preparations. We analyzed the replicates for: (i) same-day meals; and, (ii) meals on different days. For the same day, in the breakfast and snack meals, we checked the following preparations: (i) fruit; (ii) drink; and, (iii) cereal. For different days, in the lunch meal, we checked the following preparations: (i) entree; (ii) side dish; and, (iii) main dish.

- Cost limit: The Government transfers one value per school day for each student according to the school level. Thus, the cost of the menu has to be less than the pre-defined cost.

- Nutritional Error limit: Each day, each student has a nutritional needs according to their age group. The nutritional error constraint assures that no student will have a nutritional needs deviating from the ones presented in Table 1 .

Although the MPP has a combinatorial nature, it is not modeled as such, because the variables are continuous. The problem is classified as bi-objective minimization problem and is given by:

$$
\begin{aligned}
& \text { Minimize } \mathcal{F}_{1}=\text { nutritional error } \\
& \text { Minimize } \mathcal{F}_{2}=\text { cost } \\
& \text { s.a: } \quad\left\{\begin{array}{l}
\text { Color Constraint } \\
\text { Consistency Constraint } \\
\text { Variety Constraint } \\
\text { Cost Limit Constraint } \\
\text { Nutritional Error Limit Constraint }
\end{array}\right.
\end{aligned}
$$

As said previously, the objective function $\mathcal{F}_{1}$ determines how close the nutritional value is from the reference value and objective function $\mathcal{F}_{2}$ determines the cost of the menu.

Figure 1 represents a possible solution representing a menu. Each menu is defined for some days, and for each day, we have meals that are composed by preparations. The meals and preparations are represented by lists (Figure 1(a). In the integer representation (Figure 1(b) , each type of meal receives a number: (i) Breakfast (1), (ii) Lunch (2); and, (iii) Snack (3). The preparations are also represented by numbers and these numbers refer to the position of the list 
according to the type of preparation. Consider the last position of the list 1 that corresponds to breakfast in Figure 1(b) the number 11 refers to index of a list of fruits with eleven options. In this case, this index represents a pear (Figure 1(c) . For each type of preparation there is a list with several preparations.

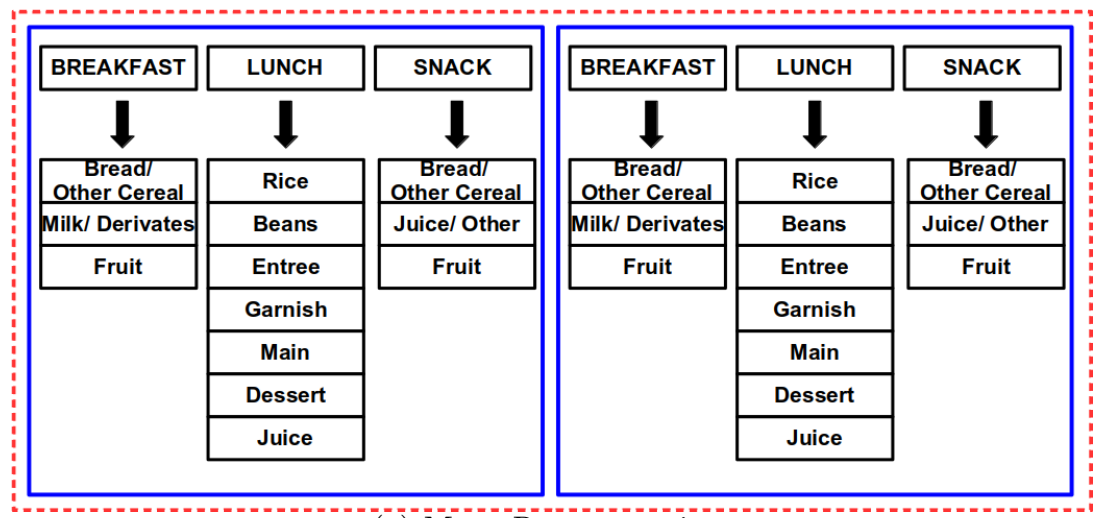

(a) Menu Representation

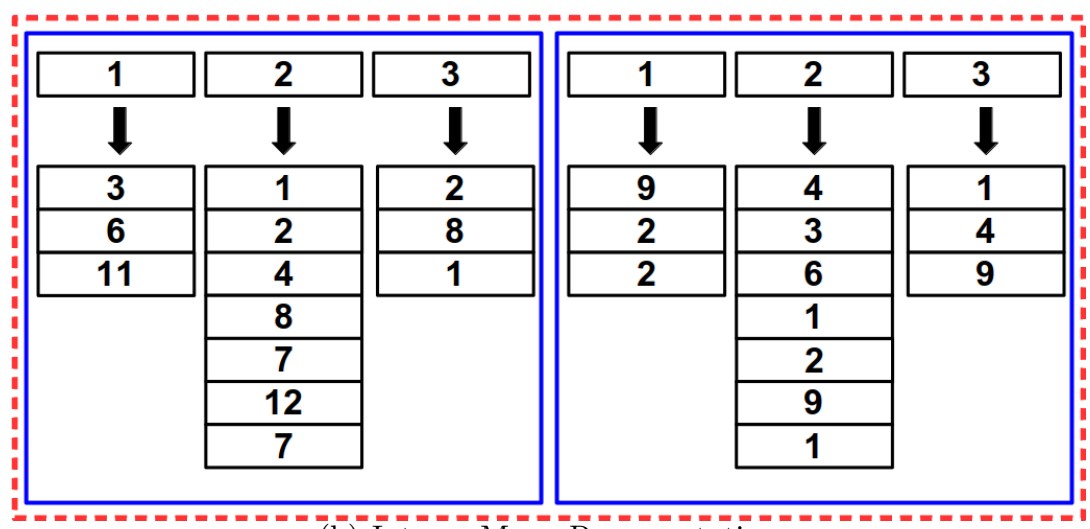

(b) Integer Menu Representation

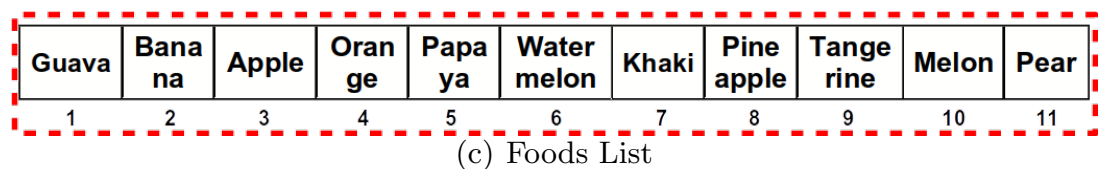

Figure 1: Representation of a two-day menu 


\section{Algorithm}

The Menu Planning Problem (MPP) is characterized as a bi-objective problem. The problem is transformed into a mono-objective problem through a weightedsum method and Genetic Algorithms are used to solve the problem. The weighted sum method is used not only to provide multiple solution points by varying the weights consistently, but also to provide a single solution point that reflects a priori preferences incorporated by the user.

The mono-objective problem can then be defined by Equation 2 .

$$
\min _{x \in \mathcal{F}_{x}} \sum_{i=1}^{q} \lambda_{i} f_{i}(x)
$$

in which $\lambda_{i}, \quad i=1, \cdots, q$ represents the weights for each objective function,

$\sum_{i=1}^{q} \lambda_{i}=1, \lambda_{i} \geq 0, \mathcal{F}_{x}$ is the feasible set for the problem and $q$ is the number of objective functions $\left(f_{i}(x)\right)$.

To solve the MPP, we implemented a Genetic Algorithm. The initial population of our algorithm is generated randomly. Depending on the type of meal, we generated random values between zero and the size of the list belonging to that type. For example, since breakfast consists of a bread/other cereal, a fruit, and a drink, three random values are generated according to the size of each list. These values represent the position in the corresponding list. Then the content of that position is added to the breakfast list. In the same way, it happens with other types of meal.

The genetic operators, selection, crossover, and mutation, are defined as follows:

- Selection: the selection is a tournament selection in which two random individuals are chosen and, the one with the best function value is selected.

- Crossover: the crossover is performed within each type of meal, according to Figure 2, Considering the crossover of breakfast and snack, the cutoff point ranges from one to three and, lunch, the cutoff points ranges from one to seven. Thus new individuals are generated preserving the genetic inheritance of its parents.

- Mutation: the mutation chooses a random day of the menu, and for each meal of this specific day, also chooses randomly a preparation to be changed by another one of the same type (Figure 3). At each generation, every individual has a chance to mutate according to a mutation rate and the preparation only be exchanged for another of the same type. For example, one fruit can only be exchanged for another fruit. Thus, in a menu, 3 exchanges can be performed.

The constraints are handling via a penalization approach. The penalties for color, consistency and variety are added to the nutritional error. Color and consistency constraints are modeled by a linear function. The variety constraint is modeled by a quadratic function. The cost limit penalty is added to the cost function. 


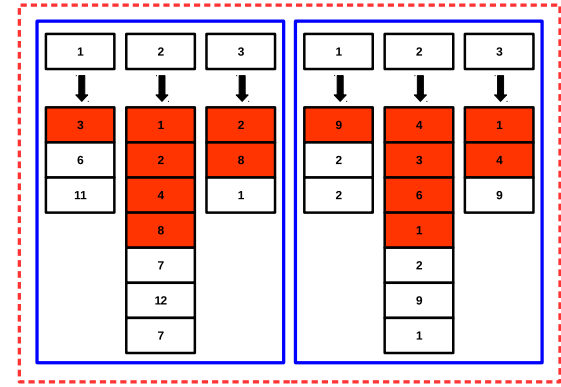

(a) Parent 1

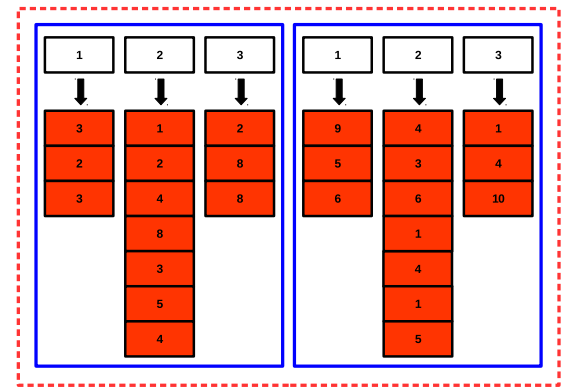

(c) Offspring 1

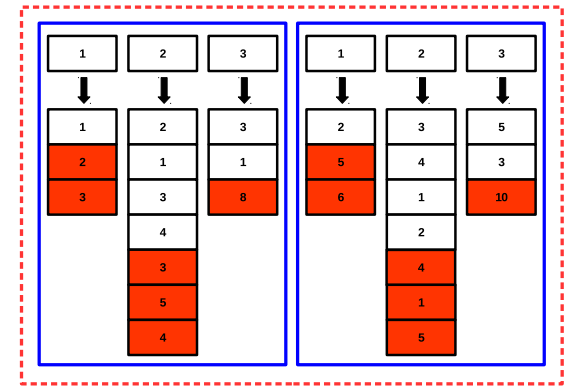

(b) Parent 2

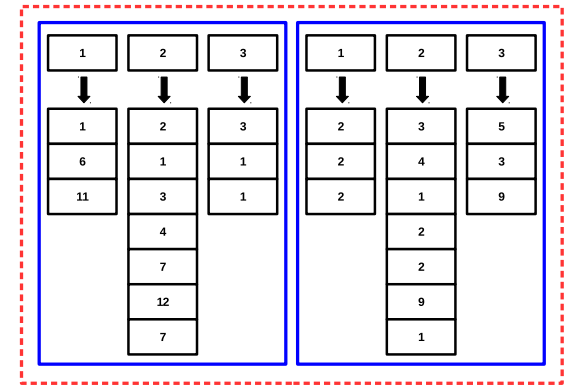

(d) Offspring 2

Figure 2: Example of crossover: Offspring 1 gets from parent 1 preparations from the first preparation of each meal to the cut-off points (1,4 and 2) and the cut-off points to the end of parent 2. Offspring 2 gets preparations from parent 2 of the first preparation of each meal to the cut-off points $(1,4$ and 2$)$ and the cut-off points to the end of parent 1.

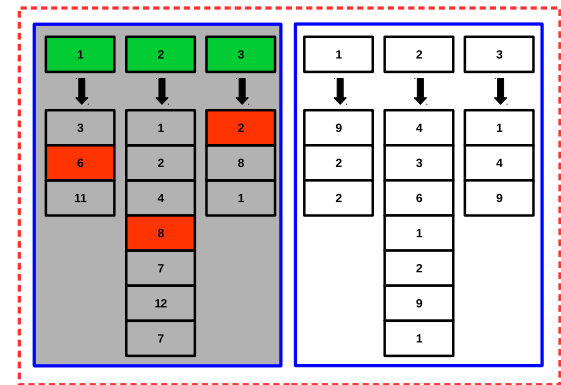

(a) Before mutation

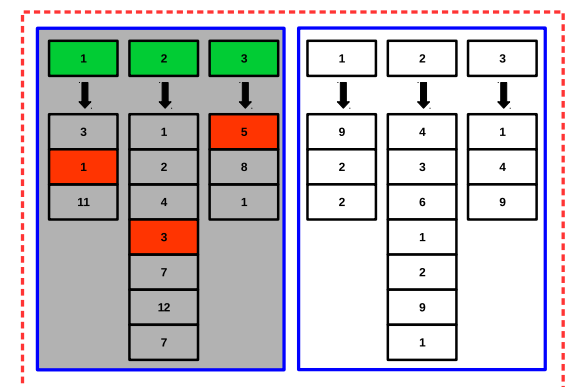

(b) After mutation

Figure 3: Example of a mutation that exchanges, in the breakfast, the preparation 6 by 1 of the milk or derivates; In the lunch, the mutation exchanges the preparation 8 by 3 of the side dish; In the snack meal, preparation 2 is exchanged by 5 of the bread/ other cereal on the first day of a 2-day menu. 


\section{Application - CardNutri}

Unlike the systems presented in Section 2, CardNutri was developed with the purpose of assisting the nutritionist responsible for the elaboration of school menus. Its objective is to elaborate collective menus minimizing the total cost and attending to the daily nutritional needs of the students, respecting the nutritional reference according to the age range provided by PNAE, variety, harmony of the culinary preparations and a financial cost limit for each type of meal.

The following tools were used to develop the system:

- Java: object-oriented interpreted programming language that has a set application programming interface (API).

- NetBeans: a free integrated development environment for the development of software in several languages, for example Java.

- API Swing: graphic library responsible for graphical system interfaces, it draws necessary components.

- MySQL: database manager system that uses the SQL language (Structured Query Language), is compatible with several programming languages.

- JFreeChart: graphics library that supports formats such as: PDF, EPS, PNG, JPEG and others.

The software works with three main bases: ingredients, culinary preparations and nutritional references. The structure of each basis and its characteristics will be presented below:

- Ingredients: items of culinary preparations. To fill this basis, the Brazilian Table of Food Composition - TACO [14, elaborated by the Nucleus of Studies and Research in Food, was adopted. It consists of 597 foods, with nutritional values for every 100 grams. As this table does not contain all the existing ingredients, CardNutri allows you to register more ingredients. The PNAE recommends that the acquisition of some food products from smallholder or family farms. The values of the products belonging to Family Agriculture were taken from the Pricing Table Practiced in the PAA - Food Procurement Program of Minas Gerais - Year 2016, of the National Supply Company - CONAB [6] and the not-listed product values were taken from the online Supermarket VipFácil [16].

Characteristics: Name; Price $(\mathrm{R} \$)(B R L-R \$ 1.00 \approx U S D-\$ 0.31)$; Nutrients: (i) Energy (kcal), (ii) Proteins (g), (iii) Carbohydrates (g), (iv) Lipids (g), (v) Fibers (g), (vi) Minerals: Calcium (mg), Iron (mg), Zinc (mg) and Magnesium (mg); Group belonging to: 1. Cereals and derivatives, 2. Vegetables, Vegetables and Derivatives, 3. Fruit and Derivatives, 4. Fats and Oils, 5. Fish and seafood, 6. Meat and dairy products, 7. Milk and Derivatives, 8. Beverages, 9. Eggs and Derivatives, 10. Sugar products, 11. Miscellaneous, 12. Other processed products, 13. Ready-to-eat food, 14. Legumes and Derivatives, 15 . Nuts and seeds.

- Culinary Preparations: individual culinary preparations. These were taken from the "School Food Menu" of Minas Gerais 4 and Goiás 3 . 
Characteristics: Name; Type: 1. Primary side dish: (i) Rice, (ii) Beans; 2. Entree, 3. Second Side dish, 4. Main dish, 5. Dessert: (i) fruits, (ii) Other 6. Drink: (i) Juice, (ii) Other; 7. Bread / other Cereal ; Color: (i) yellow, (ii) red, (iii) green, (iv) brown; Consistency: (i) Liquid / pasty, (ii) Semi-solid / solid; Ingredients.

- Nutritional References: The references were taken from Resolution 26/2013 5. The percentage of daily nutritional needs (NND) is: $20 \%$ that equals a meal for urban schools and $30 \%$ that is equivalent to a meal for indigenous or quilombola schools and $70 \%$ for a full-time school day.

Characteristics: Age: (i) 7 to 11 months, (ii) 1 to 3 years, (iii) 4 to 5 years, (iv) 6 to 10 years, (v) 11 to 15 years, (vi) 16 to 18 years, (vii) 19 a 30 years and (viii) 31 to 60 years; Percentage: (i) 20\%, (ii) $30 \%$, (iii) $70 \%$ and (iv) 100\%; Nutrients: (i) Energy (kcal), (ii) Proteins (g), (iii), Carbohydrates (g), (iv) Lipids (g), (v) Fibers (g), (vi) Minerals: Calcium (mg), Iron (mg), Zinc (mg) and Magnesium (mg).

For generating the menu, it is necessary to inform: the types of meals: 1 . Breakfast: composed of a fruit, a drink (milk or derivatives) and a bread (or other cereals); 2. Lunch: (i) Primary Side Dish: rice and beans, (ii) Entree: salad, (iii) Second Side Dish: vegetable or pasta, (iv) Main Dish: meat, (v) Dessert: pudding or fruit, (vi) Drink: juice or milk and derivatives; 3. Snack: has the same attributes of breakfast, including juice as a drink; maximum value for each type of meal; the nutritional reference to be reached and the number of days. Subsequently, all the obtained menus and its characteristics are presented. The system makes it possible to save the menus in a database as well as exporting it in pdf format.

\subsection{Graphical Interface}

The CardNutri interface is simple and user-friendly. It allows: register, change and deletes: ingredients, culinary preparations and nutritional references; generate menu; consult meals and menus; generate reports (pdf format): ingredients, culinary preparations, nutritional references, meals and menus; view system information; exit.

Figures 4.5 and 6 show the registration, alteration and exclusion screens of ingredients, culinary preparation and nutritional references. Through the "Search" button it is possible to consult all the registered items. Figure 7 illustrates the menu generation screen. It is necessary to define what types of meals and the maximum value to be paid for each of these types, the nutritional reference to be reached and the number of days, in this case corresponding to a weekly menu. The "Advanced" button corresponds to the essential settings for the technique to be applied. Observe that it is not mandatory to fill the field in since the settings are pre-defined. However, tt can be modified when needed.

Figure 8 shows the menu elaborated by the system. In this screen, you can see the number of menus generated, their composition, value and nutritional error. The graphic shows the Pareto curve of the menus found, making it easier 


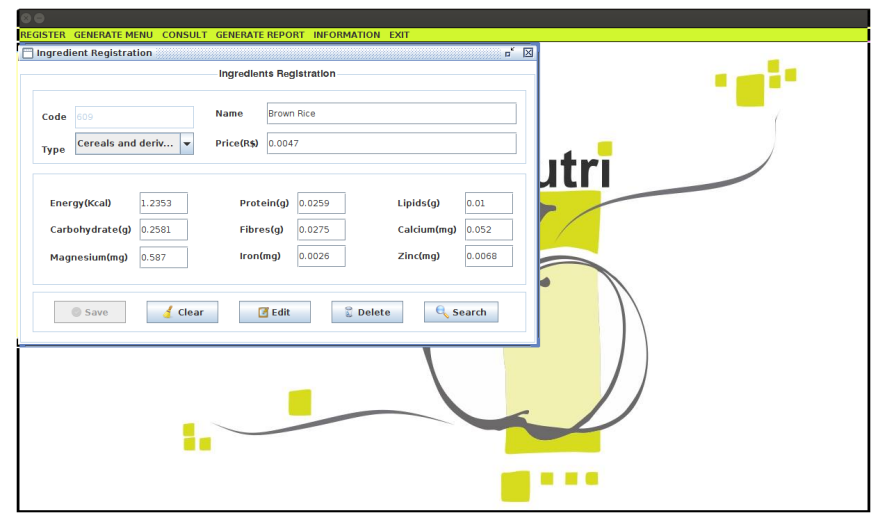

Figure 4: Registration, alteration and exclusion of ingredients

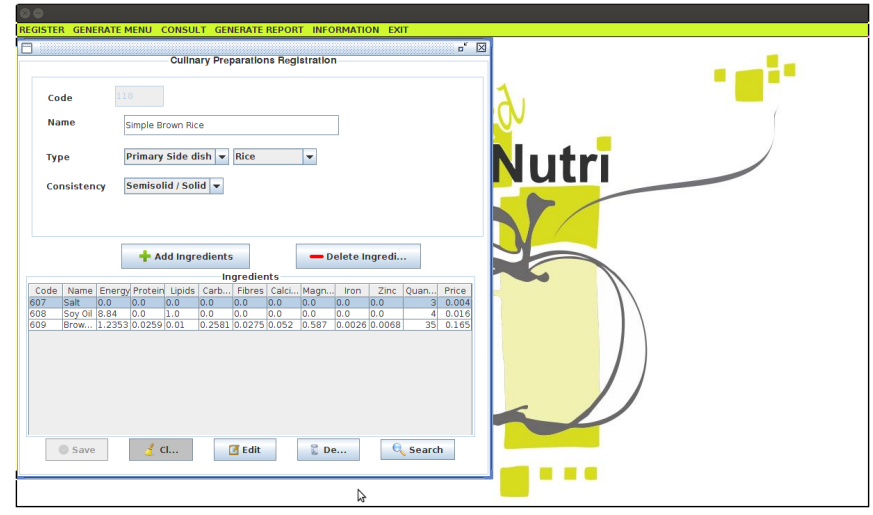

Figure 5: Registration, alteration and exclusion of culinary preparation

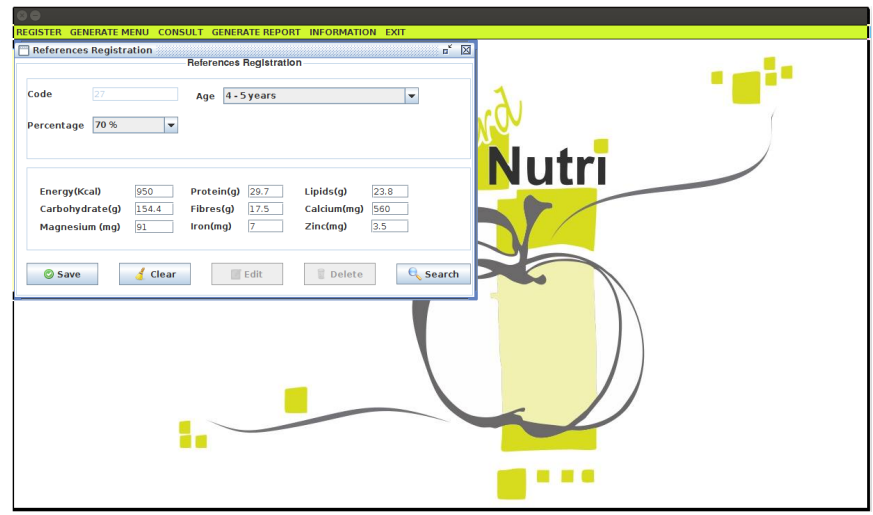

Figure 6: Registration, alteration and exclusion of nutritional reference 


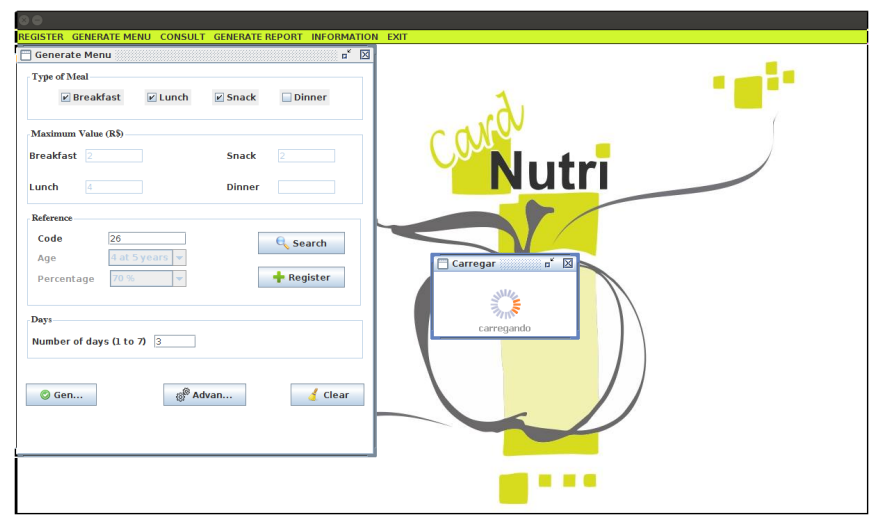

Figure 7: Menu generation screen

for the nutritionist to choose one. When you click on a point on the graphic, the corresponding weekly menu appears on the screen. The "File" menu allows you to save, and export it in a pdf format, the current menu or all menus.

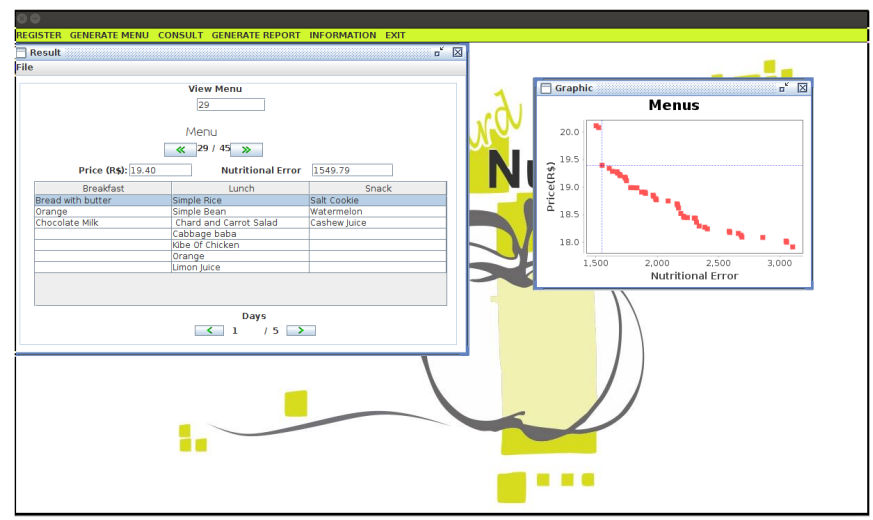

Figure 8: Menu screen elaborated by the system

\section{Results}

In this Section we evaluate the CardNutri usability and adequability of the menus.

\subsection{Solutions Evaluation}

The multiobjective optimization is characterized by returning a set of efficient solutions (Pareto set) representing a trade-off between the objective functions 
and, after that, by allowing the user to choose among the solutions, the one that best suit her/him.

The problem is solved via the Weighted-Sum method. The weight is discretized in the interval [0 1], with a step of 0.1. It is an user parameter and can be easily modified. For each weight, the GA is executed 30 times. After all GA runs, the solutions are combined and a non-dominated procedure is applied to obtain the final non-dominated set of the solutions.

The parameters for GA are: 100 individuals in the population, 1000 iterations, crossover rate equal to 0.8 , mutation rate equal to 0.05 , three meals daily in 5 days on the week. The limit values of the meals are fixed at: breakfast: $\mathrm{R} \$ 2.00$, lunch: $\mathrm{R} \$ 4.00$ and snack: $\mathrm{R} \$ 2.00$. The age group considered was 4 to 5 years, equivalent to the full-time preschool $(B R L-R \$ 1.00 \approx U S D-\$ 0.31)$.

Figure 9 shows the combined Pareto front composed of 10 different menus. Analysing this figure, it is possible to see that the algorithm found solutions with nutritional error varying, approximately, from 0.23 and 1.55 and total cost varying from $R \$ 18.91$ to $R \$ 25.32$. The range of these values complies with the pre-defined nutritional error limit and the financial cost limit. It is also worthwhile to mention that the maximum value to be paid for a 5 -day menu is $\mathrm{R} \$ 40.00$ and the 5-day menu with the highest cost $(\mathrm{R} \$ 25.32)$ represents only $63 \%$ of that value. Considering the nutritional value for a 5 -day menu, the sum of all nutrients is, according to the reference, 4434.50 and the menu which is further away from the reference has a nutritional value of 4434.76, deviating only $0.006 \%$ from that.

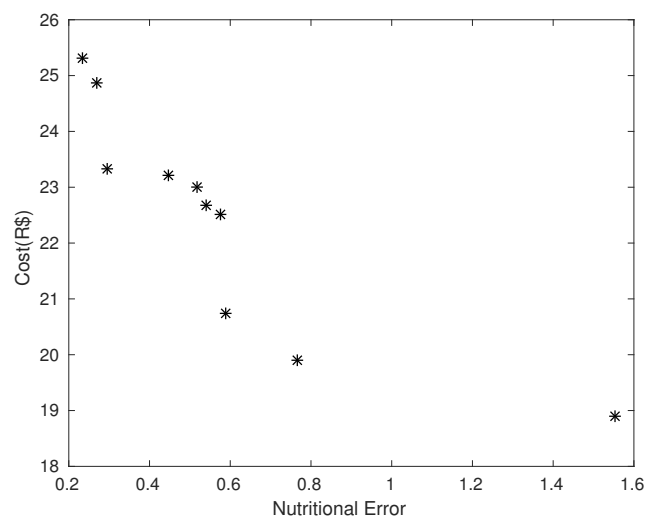

Figure 9: Combined Pareto front of menus

It can not be said that the entire set of solutions was generated. However, despite deficiencies with respect to depicting the Pareto optimal set, the results show that the obtained 5-days menus using the proposed methodology not only 
Table 2: A 5-day Menu generated by CardNutri with the respective values of nutrients

\begin{tabular}{|c|c|c|c|c|c|}
\hline Meal & Day 1 & Day 2 & Day 3 & Day 4 & Day 5 \\
\hline \multirow{3}{*}{ Breakfast } & Carrot Cake & Nutrutive Cake & Banana Cake & Bread with butter & Nutritive Cake \\
\hline & \begin{tabular}{|l|} 
Khaki \\
\end{tabular} & Banana & \begin{tabular}{|l} 
Pineapple \\
\end{tabular} & Melon & \begin{tabular}{|l|} 
Pineapple \\
\end{tabular} \\
\hline & Fruit Vitamin & Avocado Vitamin & Avocado Vitamin & Chocolate Milk & \begin{tabular}{|l|} 
Avocado \\
Vitamin \\
\end{tabular} \\
\hline \multirow{7}{*}{ Lunch } & $\begin{array}{l}\text { Greek Rice } \\
\text { with Chicken }\end{array}$ & $\begin{array}{l}\text { Rice with Broccoli } \\
\text { and Chicken }\end{array}$ & Simple Rice & $\begin{array}{l}\text { Rice with } \\
\text { Scrambled Eggs }\end{array}$ & Simple Rice \\
\hline & \begin{tabular}{|l|} 
Simple Bean \\
\end{tabular} & \begin{tabular}{|l|} 
Simple Bean \\
\end{tabular} & \begin{tabular}{|l} 
Liquid Bean \\
\end{tabular} & Simple Bean & Simple Bean \\
\hline & Braised okra & $\begin{array}{l}\text { Lettuce, Potato } \\
\text { and Carrot Salad }\end{array}$ & Watercress Salad & $\begin{array}{l}\text { Chard and } \\
\text { Carrot Salad } \\
\end{array}$ & Beet and Carrot \\
\hline & Cabbage baba & $\begin{array}{l}\text { Pumpkin Cream } \\
\text { with Green Corn }\end{array}$ & Bean soup & $\begin{array}{l}\text { Potato and Carrot } \\
\text { Creamy Soup }\end{array}$ & \begin{tabular}{|l} 
Polenta ̀̀ \\
Bolognese
\end{tabular} \\
\hline & Meatballs In Sauce & Meat quibe & Chicken Stew & Quibe Oven & Pork steak \\
\hline & Tangerina & Papaya & Caramelized Banana & Apple & Papaya \\
\hline & Grape Juice & Cashew Juice & \begin{tabular}{|l|} 
Grape Juice \\
\end{tabular} & Cashew Juice & Mango Juice \\
\hline \multirow{3}{*}{ Snack } & Banana Cake & Banana Cake & Sweet Cookie & Salt Cracker & \begin{tabular}{|l|} 
Bread with \\
tuna fish \\
\end{tabular} \\
\hline & \begin{tabular}{|l|} 
Papaya \\
\end{tabular} & \begin{tabular}{|l|} 
Papaya \\
\end{tabular} & \begin{tabular}{|l|} 
Orange \\
\end{tabular} & \begin{tabular}{|l|} 
Pineapple \\
\end{tabular} & \begin{tabular}{|l|l|} 
Apple \\
\end{tabular} \\
\hline & Cashew Juice & Grape Juice & Grape Juice & Caramelized Milk & Grape Juice \\
\hline PTN & 31.2209 & 37.9207 & 28.9064 & 35.1206 & 41.3195 \\
\hline LIP & 33.2137 & 26.3383 & 26.5924 & 25.3378 & 34.3969 \\
\hline $\mathrm{CHO}$ & 202.2888 & 194.1018 & 176.7098 & 161.5024 & 196.8131 \\
\hline Fiber & 22.3358 & 20.0105 & 20.5028 & 15.7667 & 20.3768 \\
\hline Calcium & 379.6543 & 379.8265 & 395.1736 & 459.4696 & 368.8871 \\
\hline Magnesium & 209.0066 & 217.3593 & 226.2965 & 177.8565 & 213.5767 \\
\hline Iron & 5.2033 & 5.8563 & 8.0195 & 6.1787 & 5.742 \\
\hline Zinc & 4.4691 & 5.1635 & 4.3459 & 5.3078 & 5.8109 \\
\hline $\begin{array}{l}\text { Nutrients } \\
\text { Sum }\end{array}$ & 887.3925 & 886.5769 & 886.5469 & 886.5401 & 886.923 \\
\hline
\end{tabular}

comply with the restrictions imposed by the authorities but also produce inexpensive and healthy menus.

\subsection{Software Evaluation}

CardNutri was evaluated by nine nutritionists with experience in school feeding, through a questionnaire based on the proposed Technology Acceptance Model (TAM) 7]. Participants had to use the system by performing a task list developed to help answer the questionnaire. The questionnaire contains 20 positive statements grouped into five categories: Perceived Facility, refering to the degree to which a user believes that using the system will not involve effort; Perceived Utility, refering to the degree to which a user believes that using the system will improve performance; External Variables, refering to system characteristics and training; Attitude in Relation, refering to the user's feeling of being favorable or not in relation to the use of the system; Behavioral Intention of Use, representinf the strength of intention to use the system in the future.

The questionnaire uses the five-point Likert Scale [12], which is a type of psychometric scale, where the user specifies the level of agreement or disagreement, from a statement. Each point on the scale is represented by a value in parentheses. As the affirmations are positive, the point "I fully agree" has the highest value. The levels used were: I fully disagree (1), partially disagree (2), neither agree nor I disagree (3), partially agree (4), I fully agree (5). Another technique used was Thinking Aloud [11], a strategy of thinking out loud. Participants need to express their opinions and suggestions during the evaluation or while performing some task [7]. 


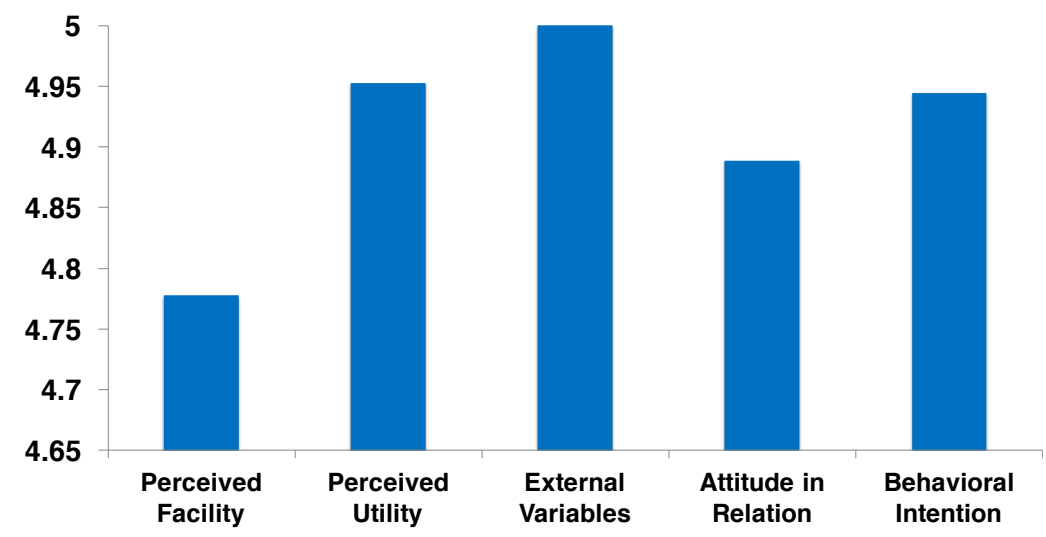

Figure 10: Means of evaluation questionnaire categories.

Figure 10 shows the average value for each category evaluated. The Figure shows that the Perceived Facility had the lowest average value (4.78). It is explained by the fact that some of the participants believe that there is a mental effort in the interaction with the system, mainly at the beginning. However, it is easy to use. Regarding Perceived Utility (average value $=4.95$ ), the system adds value to the work of the participants, increasing their performance, efficiency and productivity. Regarding the External Variables (average value = 5.0 ), they agreed that they had a good training and the explanation was clear. Regarding Attitude in Relation (average value $=4.89$ ), it would be very good to use the system instead of the manual method currently in use. And finally, on Behavioral Intention (average value $=4.94$ ), if they had access to the system, they would intend to use it.

Through the Thinking Aloud technique, all the nine nutritionists have agreed that the system reached the proposed goal of automatically developing nutritious and inexpensive menus. Despite the high rating given by nutritionists, they suggested that the system should have the option of changing a preparation after the menu has been elaborated, adding home measures, recipes, and other nutritional reference tables and calculating the value of Energy (kcal) through the macronutrients.

\section{Conclusions}

This paper presented the system of Elaboration of Nutritional Menu for School Feeding - CardNutri, developed to assist nutritionists in the automatic elaboration of menus. The goal was to plan menus automatically, in a fast and diversified manner, with low cost while satisfying the requirements established by the government via the Brazilian School Eating Programme (PNAE) guidelines. The menus were generated via a Genetic Algorithm and with the results obtained it was possible to conclude that the proposed objectives were reached and the 
restrictions established by the government were respected. CardNutri was evaluated by nutritionists with experience in school feeding, through a questionnaire based on the proposed Technology Acceptance Model. In general, results showed that the system was well-accepted and some suggestions to improve the usability were given. As future work, the nutrient-rating function to take into account the minimum and maximum macronutrient limits defined by FAO/WHO can be used to improve the results and to apply a multiobjective algorithm such as NSGA-II and compare the two approaches.

\section{Acknowledgements}

This work was partially funded by CEFET-MG, CNPq, FAPEMIG and CAPES.

\section{References}

1. Balintfy, J.: Menu planning by computer. Comunications of the ACM 7(4), 255-259 (April 1964)

2. Brazil: Food Guide for the Brazilian population. Brazil. Ministry of Health. Secretariat of Health Care. Department of Basic Attention., Brazilia, 2 edn. (2014)

3. Brazil: State Secretary of Goiás. School Meals menus., 1nd edn. (2014)

4. Brazil: State Secretary of Minas Gerais. School Meals menus., 1nd edn. (2014)

5. Brazil: Ministry of education. national fund for the development of education (fnde) pnae: School feeding (2017), http://www.fnde.gov.br/index.php/programas/ pnae

6. CONAB: National supply company (2017), http://www.conab.gov.br/

7. Davis, F.D.: Perceived usefulness, perceived ease of use, and user acceptance of information technology. MIS Quarterly 13(3), 319-340 (1989)

8. Flores, de Gpe.Cota, M., Ramárez, D., Jiménez, I.J., Raygoza, J.A., Morales, L.C., Galaviz, S., Espinoza, A., Orozco, M.E.: Pladiet: Un sistema de cómputo para el diseño de dietas individualizas utilizando algoritmos genéticos (2007)

9. Gomes, F.R.: Pro-diet: Automatic generator of personalized menus based on Genetic Algorithm. Masters dissertation, Federal University of Uberlândia, Uberlândia (2012)

10. Kahraman, A., Seven, H.A.: Healthy daily meal planner. Genetic and Evolutionary Computation Conference - Undergraduate Student Workshop (GECCO 05 UGWS) pp. 25-29 (2005)

11. Lewis, C.: Using the "Thinking Aloud" Method in Cognitive Interface Design. Research report, IBM T.J. Watson Research Center (1982)

12. Likert, R.: A Technique for the Measurement of Attitudes. No. $\mathrm{N}^{\mathrm{o}} 136-165$ in A Technique for the Measurement of Attitudes, publisher not identified (1932)

13. Nations, U.: Foof and agriculture organization of the united nations (2017), http: //www.fao.org/home/en/

14. NEPA: Nucleus of studies and researches in food. brazilian table of food composition - taco (2011), http://www.unicamp.br/nepa/taco/tabela.php?ativo= tabela

15. Sufahani, S., Ismail, Z.: A new menu planning model for malaysian secondary schools using optimization approach. Applied Mathematical Sciences 8(151), 75117518 (2014)

16. VIPFACIL: Bh online supermarket (2017), https://www.vipfacil.com.br/ 